\title{
Fenomenología de la intersubjetividad y su importancia para la comprensión de las enfermedades endógenas
}

Otto Dörr Zegers ${ }^{1}$

\section{Resumo}

Um dos traços característicos dos transtornos endógenos é a alteração das relações interpessoais. Essa condição permite a realização, nesses estados patológicos, de uma fenomenologia da intersubjetividade, que revele as modificações das condições de possibilidade da presença do outro na esquizofrenia, depressão melancólica e mania. Após rever as contribuições fundamentais da psicopatologia fenomenológica nessa área, o autor propõe uma releitura da patologia da interpessoalidade a partir da fenomenologia da intersubjetividade de Edmund Husserl. A partir dos conceitos de apresentação/presentação e retentio/presentatio/protensio, sustenta que a mania é constituída por um fracasso de apresentação. Na depressão melancólica, por sua vez, a retentio invade a protentio e paralisa a apresentação. Examina a esquizofrenia por meio do conceito de intencionalidade, defendendo que a perda desta é típica da patologia.

Palavras-chave: Fenomenologia da Intersubjetividade; Mania; Depressão Melancólica; Esquizofrenia; Fenomenologia Husserliana.

\section{Abstract}

One of the main traits of the endogenous diseases is the alteration of the interpersonal relationships. This allows the development, in these pathological states, of a phenomenology of the intersubjectivity, revealing the conditions of possibility of the otherness in schizophrenia, melancholic depression and mania. After reviewing the fundamental contributions in phenomenological psychopathology in the field, the author proposes a reappraisal of the pathology of the intersubjectivity following Edmund Husserl's phenomenology of the intersubjectivity. Taking the concepts of appresentation/presentation and retentio/presentatio/protensio, supports that mania is constituted by a flaw in the appresentation; in the melancholic depression, by its turn, retentio invades protentio

\footnotetext{
${ }^{1}$ Profesor de Psiquiatría de la Universidad de Chile y de la Universidad Diego Portales.
} 
and paralyses appresentation. The author examines schizophrenia through the concept of intentionality, and affirms that the lack of it is typical of the pathology.

Keywords: Phenomenology of Intersubjectivity; Mania; Melancholic Depression, Schizophrenia; Husserlian Phenomenology.

\section{Introducción}

Nadie podría discutir que uno de los rasgos centrales de ese conjunto de enfermedades que llamamos esquizofrenia es la alteración a nivel de la relación interpersonal o, expresado en términos más abstractos, la alteración de la interpersonalidad $\mathrm{o}$, dicho en forma más genérica, de la intersubjetividad. El autismo, considerado por Bleuler (1911) como uno de los síntomas fundamentales de la enfermedad y esa dificultad de contacto, perceptible ya años antes de manifestarse ella, tema sobre el cual Minkowski (1927) escribiera páginas memorables, son sólo dos ejemplos de la trascendencia de esta perturbación, si es que queremos acercarnos a la esencia de esta enfermedad. Porque ocurre que también otros síntomas de la esquizofrenia pueden ser vistos desde la misma perspectiva. Pensemos en el síndrome paranoídeo, por ejemplo, donde el otro aparece como imbuido de poderes mágicos, por medio de los cuales el paciente es perseguido, asediado y por último, invadido en la intimidad de su propia conciencia misma a través del síndrome de influencia. Pero también las alucinaciones auditivas, que a una mirada superficial pueden aparecer como un síntoma tan "objetivo", que podría incluso atribuirse a alteraciones de la compleja función de la audición, pueden ser vistas como una peculiarísima perturbación de la relación con el otro, transformado aquí en un ser anónimo, atemporal, agresivo e invasor. Y lo afirmado hasta acá no vale sólo para las formas paranoídeas, hebefrénicas y catatónicas, donde los síntomas enumerados se presentan, aunque en distintas combinaciones, con mayor frecuencia. En un trabajo de 1965 (DörrZegers, 1965) y vuelto a publicar como capítulo del libro Psiquiatría Antropológica (Dörr-Zegers, 1995), pudimos demostrar cómo a la base de las formas hipocondríacas de la esquizofrenia, esas centradas fundamentalmente en el propio cuerpo y habitualmente clasificadas dentro de las formas "simples" de la enfermedad, se encontraba también una alteración de la interpersonalidad, de la relación con el otro, con el tú. 
El paciente que analizamos en aquella oportunidad, que era un estudiante muy inteligente y tenía una gran capacidad de introspección, escribía en su diario en el período anterior a la irrupción de la psicosis cosas tan impresionantes como las siguientes: "Mi falla es el amor a mí mismo. Aún no he encontrado el camino hacia el tú. Fuera de un corto período en Waldheim (un colegio), yo siempre he estado encerrado en mí mismo, sordo frente al mundo exterior. Lo que tengo que lograr es una inclinación desinteresada hacia lo exterior a mí... Quiero aventurarme hacia otra fase, la del amor al prójimo." Y en el comienzo de la psicosis decía cosas tan extrañas, como que su cerebro estaba atrofiado y se había desviado hacia el tórax, pero que en todo caso no era capaz de llenar el cráneo. Su propio cuerpo se le había hecho extraño; para poder asegurarse de que sus brazos y piernas eran propios, los observaba, los movía y los mordía. Sentía que su cuerpo era plano y que las cosas se le venían encima y penetraban en él. Y en su diario de vida anota: “El mundo está muy alejado, sin significado y vacío. Por ejemplo, si yo observo una granja, veo con gran exactitud la casa y las personas, pero sólo como algo material. Más allá de eso todo es pobre, desierto y sin significado." Y más adelante anota: "El mundo llega directamente a mí, no hay distancia entre el mundo y yo. Y eso vale tanto para las personas como para las cosas. La cercanía llega hasta la identificación con las cosas y las personas. Puede suceder incluso que en los peores estados me considere por momentos como el otro, como aquel a quien yo miro."

Él mismo describe también esta dificultad que tenía de llegar a la otra persona, en este caso a una muchacha de quien se había enamorado: "Fue algo muy triste. Cuando se produjo el encuentro con ella, hubiera necesitado sólo saludarla, pero en ese momento me cerré completamente, como una navaja. Todavía la veo venir hacia mí. De repente todo se hizo nebuloso, como si lo hubieran borrado. Yo comencé a temblar, me di vuelta y seguí de largo." Esta incapacidad de encuentro se va haciendo más y más marcada, hasta el punto que Federico no está en condiciones siquiera de reconocer a las personas en cuanto sujetos individuales y así es como escribe en su diario: "Hace tiempo que ya no puedo acordarme de lo individual propio de los rostros de personas conocidas. Cuando los miro sólo les veo las piernas."

Walter von Baeyer (1955) definió con extraordinaria agudeza las características de este encuentro interpersonal deformado de los esquizofrénicos, pero basándose fundamentalmente en casos de enfermos paranoídeos. Esta forma de encuentro se caracterizaría por la falta de reciprocidad, la anonimización y la mediatización. En el delirio de amor, por ejemplo, sobre todo cuando adquiere características alucinatorias, el paciente no puede defenderse de las voces 
amorosas o de las alucinaciones cenestésicas vinculadas a la esfera sexual, vale decir, es un encuentro sin reciprocidad. Es corriente que ese tú invasor, que en un comienzo tiene un nombre, vaya perdiendo su individualidad, para hacerse colectivo. Son todos los jóvenes de la ciudad o aún todos los hombres, los que acosan a la paciente. Por último, ese contacto con el otro alucinado, que en un primer momento es directo, se mediatiza, a través de aparatos como radios, de televisores, de transmisores, etc. Nosotros pudimos demostrar que un fenómeno semejante ocurría también en esta forma en cierto modo polar respecto a la esquizofrenia paranoídea, que representa la esquizofrenia simple con características cenestésicas. La diferencia entre una y otra estriba sólo en que en la segunda el otro invasor aparece más y más como un mero cuerpo despersonalizado.

En el caso de la depresión y aún cuando las quejas de los pacientes se refieran por lo general no al otro, sino al sentimiento de sí mismo y a cambios en el propio cuerpo, también se podría concebir la alteración fundamental desde la perspectiva de lo interpersonal. Se podrían distinguir dos formas de depresión esencialmente diferentes, hasta el punto de haberse discutido más de alguna vez si no se trata de entidades nosológicas distintas. Me refiero a las formas delirantes y a las no delirantes de depresión. La segunda, que es la más frecuente, muestra como fenómenos fundamentales, como lo hemos desarrollado en otros trabajos (DörrZegers 1979, 1993, 1995, Dörr-Zegers \& Tellenbach, 1980), el cambio negativo de la experiencia del cuerpo (decaimiento, desinterés, angustia, dolores, sensación de frío, etc.), el "no-poder" (concentrarse, decidirse, actuar, sentir placer, etc.) y la alteración, inversión o suspensión de los ritmos vitales. En la primera, en cambio, la sintomatología está comandada por el delirio (de culpa, ruina o enfermedad), aún cuando en un segundo plano puedan encontrarse también los mismos fenómenos propios de la depresión clásica. Las diferencias no sólo se refieren a la sintomatología, sino también a la personalidad previa, la biografía y las situaciones desencadenantes. Y sin embargo y desde la perspectiva del encuentro, ambas tienen algo en común: el progresivo desinterés en el otro y su reemplazo por el tema predominante: el cuerpo en una, el delirio en la otra. Es frecuente observar cómo los pacientes depresivos no pueden desprenderse de sus síntomas corporales, ya sea la falta de fuerzas, el decaimiento, la angustia, el insomnio, los dolores, etc. En un antiguo trabajo empírico-fenomenológico sobre el síndrome depresivo nuclear o melancólico (Dörr-Zegers, 1997, Dörr-Zegers, Enríquez \& Jara, 1971) encontramos que los síntomas corporales estaban presentes en el $100 \%$ de los episodios depresivos de 55 pacientes que en un período de cinco años habían estado internados por este motivo en el Hospital Psiquiátrico de Concepción. En 
cierto modo el paciente depresivo se torna inalcanzable, detrás de ese cuerpo que lo ocupa por entero, pero al mismo tiempo se hace inmodificable. Kraus (1998) describe esa particular irritación que provoca el paciente depresivo en el examinador, al sentirse éste imposibilitado de influir de alguna manera sobre el estado de ánimo de estos enfermos. Kranz (1972) sostenía que los depresivos eran "más autistas que los esquizofrénicos", apuntando con ello al mismo fenómeno que hemos venido señalando.

Este carácter inalcanzable e incomprensible del ánimo depresivo (a diferencia de lo que ocurre con la tristeza) adquiere su máxima expresión en el estupor depresivo. En un trabajo anterior (Dörr-Zegers \& Tellenbach, 1980) intentamos hacer una fenomenología de la corporalidad depresiva, partiendo justamente de la experiencia que tiene el examinador frente a un caso de estupor, estudio que continuamos después desarrollando en otra publicación (Dörr-Zegers, 1993). En esta última decíamos: "En cierto modo ella no está frente a nosotros, por cuanto no se produce entre ella y yo esa natural tensión polarizada que ocurre en los encuentros interpersonales y que los griegos llamaban antikry" (p. 25). Y más adelante explicábamos esto con las siguientes palabras:

La paciente permanece insensible a todos mis intentos de llevarla a algo así como una contraposición, que se me oponga como un otro y es curioso, pero el sentimiento que me va provocando se aleja más y más de la pena o la compasión... Esa impresión de lo desagradable y ajeno resulta de la experiencia de un vacío, allí donde yo estaba preparado para encontrarme con una persona de espíritu vivo y diferente, más o menos comunicativa, familiar, abierta, simpática, o lo que sea. (p. 25)

La perturbación de la interpersonalidad en los pacientes depresivos no delirantes se observa en todos los grados de esta enfermedad, desde aquellos en que se manifiesta sólo a través de la preocupación casi única por un síntoma, casi siempre de tipo corporal, como el insomnio o el estreñimiento, hasta aquellos donde la invasión del espíritu por el cuerpo es total (proceso de "cosificación", op. cit., 1993) como es el caso del estupor. En las depresiones delirantes volvemos a encontrarnos con esa cerrazón del paciente frente al encuentro interpersonal, dominado como está por esta idea central que constituye su delirio. También hemos trabajado este tema a propósito de una paciente con un síndrome de Cotard (Dörr-Zegers, 2002). En este caso no era sólo la fuerte impresión del examinador del carácter inaccesible de la persona de la paciente, sino que ella misma, que era 
muy inteligente, era capaz de describir en forma insuperable este encierro en su cuerpo y su incapacidad de llegar al otro:

Lo que pasa conmigo es que tengo todo muerto; digamos, que estoy muerta, que estoy en estado vegetal de mi cabeza a los pies. No tengo tacto, ni olfato, ni gusto por las comidas. Mi cuerpo es tan liviano que es como si no existiera... Cuando tomo a mis hijos en los brazos, no los siento... Si mis hijas supieran que están queriendo a una mamá artificial... Yo no siento el contacto directo con las cosas, tampoco con los demás... Ahora, cuando hablo, las palabras salen automáticamente de mí, pero las de los otros no entran en mi cabeza... Lo único que quiero es morirme, pero sigo viviendo porque, al parecer, el corazón late, aunque tampoco lo siento... (Dörr-Zegers, 2002, p. 5)

¿Y qué ocurre con la manía? Lo más llamativo en ellos es sin duda la euforia, la hipervigilia, la hiperactividad, el pensamiento ideofugal y el insomnio. Este ser atraído por todos los estímulos, incluyendo naturalmente las personas que tiene cerca, hace pensar a primera vista que el maníaco tiene un exceso de capacidad de encuentro y comunicación con el otro y de hecho, se emplea como criterio diagnóstico diferencial con respecto a las psicosis esquizofrénicas la "sintonía" del paciente, la capacidad de transmitir al otro sus sentimientos y emociones. Sin embargo, si observamos con mayor atención, vamos a poder constatar también aquí una profunda alteración a nivel de la interpersonalidad. Hay en estos pacientes un tratar al otro con excesiva confianza y una pérdida de la distancia social hasta el extremo de la irrespetuosidad, que no puede sino llamar profundamente la atención. Ese tomar al otro "por el lado flaco", como dice Binswanger (1960), ese reírse de los defectos de los demás, así como el señalar con risas inadecuadas cualquier disonancia en el entorno, son todos ejemplos de cómo el paciente maníaco es incapaz de constituir una armónica y auténtica relación con el otro. Un ejemplo personal y reciente: una paciente bipolar de 70 años, de alto nivel socio-económico y cultural, después de salir de una depresión profunda cicló a una leve hipomanía, perceptible por la familia sólo en su irritabilidad y en el despertar temprano. En medio de la entrevista en que yo compruebo su mirada brillante, su mayor movilidad y un maquillaje algo excesivo, ella observa: "El lápiz que usted está usando es demasiado ordinario. Yo le voy a regalar uno mejor para Navidad." Ahora, la observación era justa: el lápiz que yo uso habitualmente se me había extraviado pocos días antes en el aeropuerto y mientras encontraba el tiempo para comprarme otro, estaba usando uno común y corriente. No obstante, nadie dudaría en considerar que los dichos de la paciente son de algún modo 
inadecuados. La pregunta sería, entonces, ¿qué es lo que está fallando aquí? Porque sus palabras habrían sido justificadas quizás si yo hubiera sido su hijo o su hermano, pero soy su médico y estamos involucrados en esa situación tan seria y trascendente, como es la relación médico-paciente.

Un muy buen ejemplo de este equivocarse en la apreciación de lo que se debe hacer o decir en una situación social determinada, de este verdadero fracaso en la constitución de una adecuada relación con el otro, lo relata Binswanger (1960) en su libro Melancholie und Manie: una paciente maníaca se arranca de la clínica donde está hospitalizada, vaga por las calles y entra a una iglesia donde en ese momento tiene lugar un servicio religioso y un organista está tocando. Ella sube hasta donde está el órgano, felicita al organista por lo bien que toca y le pide majaderamente que por favor le empiece a dar clases lo antes posible. Un ejemplo muy simple, pero ¡qué decidor! Nadie podría dejar de sentir que aquí también hay algo inadecuado, que no corresponde, que se está desconociendo el verdadero contexto de la situación.

\section{Teoría husserliana de la intersubjetividad}

Hasta aquí y a través de observaciones clínicas hemos sugerido la hipótesis de que detrás de estas misteriosas enfermedades endógenas, la esquizofrenia, la melancolía y la manía, se esconde una profunda alteración de la interpersonalidad. Ahora la tarea es interpretar estos hechos a la luz de la fenomenología de la intersubjetividad de Edmund Husserl (1963). Para ello intentaremos resumir los elementos más importantes de esta teoría. Sería, por cierto, imposible reproducir aquí todo el contenido de la quinta meditación cartesiana, donde se encuentra lo fundamental de la teoría husserliana de la intersubjetividad, pero un concepto fundamental para la concepción fenomenológica de la intersubjetividad es la "apresentación". Husserl llama "apresentación" primero a lo que se agrega a la

presentación corporal del otro y con lo que se fusiona luego en una unidad, permitiendo la experiencia del alter ego. Se trata aquí de una experiencia de la esencia de un ente real que no es el mío propio, pero que no se agrega a lo mío propio como parte integrante, o sea que trasciende totalmente a mi propio ser, aunque "sólo puede adquirir sentido y justificación desde él". Se trata entonces de un caso especial del gran problema husserliano, de mostrar cómo lo trascendente en general puede volverse "inmanentemente trascendente". La solución de este 
problema se llama, como ya sabemos, constitución, constitución tanto de la objetividad temporal, del mundo propio y del común, como también del alter ego, del otro y su mundo.

Para aclarar la cuestión de la constitución del alter ego se necesita antes que nada aclarar el problema de la constitución del ego y de mi mundo propio. La pregunta que dirige Husserl es la siguiente: ¿Qué es lo propio para mí en cuanto puro sí mismo y en esa medida no permutable y qué es lo que, a pesar de este algo no permutable, es comunicable? Husserl llama a la primera esfera, esfera original o primordial. Es la esfera de mi fluir vital interno, de mi propia y no permutable "historia vital interna" (Binswanger, 1947). En el orden de la constitución de un mundo externo ajeno al yo, ese fluir vital interno es en sí mismo el primer mundo y por eso llamado primordial. Mi cuerpo (o cuerpo-sujeto) es el único soma (o cuerpo-objeto) que está y puede ser constituido originariamente como cuerpo o corporalidad. Allí donde un cuerpo está hecho en forma semejante al mío es claro que tiene que recibir su sentido desde mi cuerpo. La pregunta que se plantea entonces es la siguiente: ¿Qué es lo que hace que de ese cuerpo resulte el cuerpo del otro y no un segundo cuerpo propio, un duplicado? La respuesta ya la sabemos: es el hecho que nada de ese otro cuerpo puede ser integrado a mi propio flujo vital en cuanto mío.

El problema se agudiza si se plantea la pregunta, ¿cómo es eso que, a pesar de la imposibilidad de una actualización del cuerpo ajeno y con ello, de la psique ajena, pueda llegarse a una experiencia del otro? La respuesta a esta pregunta representa uno de los logros más geniales de Husserl y demuestra claramente la estructuración constitutiva de la experiencia del otro desde la presentación o percepción de un cuerpo real, vale decir, algo perteneciente a la naturaleza, hasta la apercepción del mismo en cuanto cuerpo-sujeto (Leib) y luego la "apresentación" del mismo como alter ego. Las tres intencionalidades se encuentran entrelazadas del modo más íntimo en la percepción del otro. En este entrelazamiento, el cuerpo del otro y el yo del otro aparecen dados en el modo de una experiencia unitaria y trascendente. Por cuanto cada experiencia está dispuesta sobre otras experiencias que llenan y confirman los horizontes "apresentados". El resultado es entonces que la "apresentación" "presupone un núcleo de presentación" o, en forma más exacta, que ella es una actualización asociada con la auténtica percepción (del cuerpo extraño). Ambas, percepción y actualización, están aquí en la comunidad funcional de una percepción que se presenta y "apresenta" al mismo tiempo, creando la conciencia de su auto-existencia para todo el objeto. Es así como mi ego constituye en sí mismo al otro ego. 
Después de haber mostrado la constitución de la percepción del otro sobre la base de la intersubjetividad, pasaremos a explicar el problema de la intersubjetividad o de la constitución del mundo común. Es corriente que el lego use la expresión: "El enfermo mental vive en un mundo distinto que nosotros", pero ni aún para nosotros, los psiquiatras, queda claro qué sentido científico tiene esta expresión ni tampoco en qué consiste la anomalía de la constitución de ese ser distinto del mundo de los enfermos mentales. Szilasi (1959) ha mostrado con toda claridad que la constitución del mundo común también es realizada a través de la "apresentación" y que esta acción está fundamentada en la presentación con las retenciones y protensiones correspondientes. Las referencias intencionales que conducen a la "apresentación" son por una parte de tipo objetivo y por otra, de tipo histórico biográfico. Mientras las presentaciones, como ya sabemos, pertenecen a nuestro flujo vital con sus momentos inmanente-trascendentes en el sentido del mundo primordinal de Husserl, mi Ego constituye un trozo de mundo común a través de lo "apresentado". Para ello da Szilasi $(1959$, p. 109) un ejemplo muy clarificador:

Yo en cuanto Yo-vida (Lebens-Ich) me soy presente en todos los actos de la conciencia. Pero yo también me puedo ser presente acompañado de "apresentaciones". Por ejemplo, en este momento me soy presente a mí mismo, pero al mismo tiempo, me soy "apresente" como profesor de filosofía que está dando una clase. Para Ustedes, como auditores, yo estoy presente de un modo distinto al que estoy para mí mismo, pero en la misma "apresentación", a saber, como alguien que está dando una clase. Nuestras presentaciones son diferentes, pero están acompañadas de la misma "apresentación". Lo "apresentado" idénticamente por ustedes, que son para mí alter egos ajenos y por mí, que soy para ustedes un alter ego ajeno, es lo común y eso es lo que constituye el mundo común (lo objetivamente trascendente). Mientras ustedes se concentran en el contenido de la clase, éste constituye el tema de su reflexión actual. Para ustedes está presente y constituyendo en múltiples retenciones y protensiones el mundo propio para ustedes en su propio fluir de sus respectivas vidas. En este proceso ustedes están "apresentes" para sí mismos como auditores o estudiantes. En la misma calidad están ustedes también "apresentes" para mí (pero no presentes como Egos propios, por cuanto la vida psíquica de ustedes me es inaccesible). 


\section{Fenomenología de la intersubjetividad y psicosis maníaca}

Remitámonos al ejemplo de Binswanger de la paciente maníaca que ingresa a una iglesia donde se desarrolla un servicio religioso e interrumpe al organista. Para el lego, la actitud de la paciente puede ser juzgada como inadecuada, desconsiderada, incomprensible, etc. El psiquiatra hablará aquí de facilitación del actuar y de pérdida de inhibiciones. Pero ninguno de estos dos juicios nos da razón sobre lo que está realmente sucediendo acá. Así como la medicina somática tiene la teoría del organismo como marco dentro del cual puede ella "medir" las desviaciones con respecto a la norma, así también tendría que poder hacerlo la psiquiatría. Pero esta ciencia básica no está suficientemente desarrollada y a nuestro entender no cabe sino que ese lugar lo ocupe, siguiendo a Binswanger, la fenomenología de la conciencia intencional.

También el organista y la paciente que lo importuna tienen presente algo distinto, a saber, su propio flujo vital interno, con su propio mundo primordial. Pero estas distintas presentaciones no están acompañadas aquí de la misma "apresentación". El organista está presente para sí como el fluir de contenidos de conciencia, las sensaciones provenientes de su cuerpo, entre otras las de sus dedos tocando el órgano. Estas presentaciones están acompañadas de la "apresentación" de que él es un organista que ha sido contratado para tocar en el servicio religioso y esa misma "apresentación" la comparte con la comunidad que asiste a la iglesia. La paciente, en cambio, no comparte esta "apresentación" y de ningún modo "toma parte" en ella. Como presencia en su percepción (de la paciente) sólo está un organista tocando, pero ella no "apresenta" que se trata de un concierto en el marco de un servicio religioso. Ahora bien, la paciente, después de retirarse de la iglesia, entró a una cancha de fútbol donde unos muchachos jugaban un partido, causando entre ellos, además de la perplejidad, rabia e hilaridad. Tampoco aquí logró tener la misma "apresentación" que ellos y constituir así un mundo en común. El que haya cambiado bruscamente de un escenario a otro no es una simple consecuencia de su hiperactividad patológica, sino del hecho de vivir en meras presencias aisladas, sin una conexión entre ellas, inexplicables por ende desde su biografía, porque esta paciente no ha podido ordenar esas presencias en el continuum de su historia vital interna. También significa esto que han fracasado las protensiones y las retenciones. Sólo anticipando el futuro y reteniendo el pasado podemos actualizar el presente. Al aflojarse esta interacción perfecta entre pasado, presente y futuro, entre retentio, protentio y presentatio, la vida misma se transforma en la conciencia de fragmentos de mundo sin orden ni concierto. 
De la teoría de Husserl expuesta al principio se desprende que si se fracasa en la constitución del alter ego, si no se es capaz de una verdadera comprensión del otro, también fracasa la constitución de sí mismo, del ego propio. La paciente de Binswanger es incapaz de captar "apresentativamente" el sentido del organista (en su contexto), porque ella misma no puede experimentarse "apresentativamente" como un ego. Tanto en la iglesia como en la cancha de fútbol, la paciente está presente para sí misma en su mundo primordial (o flujo vital interno), pero no logra "apresentarse" como la señora Fulana de Tal, como esposa y madre de cuatro hijos, como persona bien educada, dama de la sociedad y con mayor razón aún, como paciente que huyó de una clínica psiquiátrica. Y esto sucede porque su vida transcurre de presencia en presencia, desconectada de retenciones y protensiones, vale decir, a causa del total derrumbe de la temporalización. Esto explica el por qué el maníaco fracasa en la constitución del mundo común, aún cuando y a diferencia del depresivo, se observa en él la tendencia a volcarse hacia los demás. Pero este dirigirse hacia los otros no está dirigido hacia una auténtica comunicación, sino más bien es la consecuencia de una huída de sí mismo, del propio yo y al alejarse del propio yo el otro pierde el carácter fenomenológico de alter ego y deviene un allius, un ser extraño y ajeno. No es muy diferente lo que le ocurre a mi paciente, la que, desconociendo la situación de entrevista psiquiátrica y la peculiaridad de la relación médico-paciente, me hace un reproche que sólo cabría en el contexto de la mayor intimidad familiar: "Usted está usando un lápiz demasiado ordinario; yo le voy a regalar uno mejor para Navidad".

\section{Constitución de la temporalidad objetiva y depresión melancólica}

El fluir de mi vida interna es, por cierto, inseparable de la temporalidad originaria o primordial, con respecto a la cual el tiempo objetivo o del reloj es sólo un derivado. $Y$ esa temporalidad originaria se da como el permanente entrelazamiento de tres instancias que Husserl llama retentio (pasado), protentio (futuro) y presentatio (presente). Para comprender el papel fundamental que juegan estas instancias temporales tanto en la constitución del propio yo (Ego) como del otro (alter Ego) baste pensar en el hecho que cada persona que está hablando en un momento dado (presentatio) no podría decir lo que está diciendo sin saber exactamente lo que ya dijo, vale decir, sin retener el pasado (retentio) y lo que va a decir, con otras palabras, sin anticipar el futuro (protentio). Y esto no vale sólo para 
el caso de un discurso o de una conferencia, sino para cualquier conversación, por simple que ella sea.

Ahora bien, en la depresión endógena, también llamada melancolía o depresión melancólica, la alteración de la interpersonalidad no se encuentra en el fracaso de la "apresentación", como vimos que ocurría en la manía. Por el contrario, sabemos cuán normadas y respetuosas son las personalidades proclives a la depresión, según lo intuyera tempranamente Tellenbach (1961/1983) y lo demostrara von Zerssen a través de una serie de investigaciones empíricas (1969, 1970, 1982), de modo que ellos jamás desconocerían el sentido del contexto situacional en la relación con el otro. Pero en un comienzo describimos de qué manera el paciente depresivo se encerraba en su cuerpo o en su delirio, tornándose inalcanzable para el otro. ¿Entonces, qué es lo que falla en la relación del depresivo con el otro, desde un punto de vista fenomenológico? En primer lugar está ese encierro en el cuerpo y esa inaccesibilidad que mencionábamos en la introducción. Pero hay otros dos fenómenos muy característicos que podrían ser interpretados también desde la intersubjetividad y que han sido destacados por Binswanger (1960). Se trata de los auto-reproches y de las ideas deliroides depresivas. Cuando un paciente depresivo se reprocha, por ejemplo, "si yo no hubiera vendido la casa, entonces no me habría arruinado..." o "si yo no hubiera organizado ese paseo donde ocurrió el accidente, Fulano no habría muerto...", etc., lo que está haciendo es colocar en el pasado, que de por sí es inamovible, posibilidades vacías. Dicho con otras palabras, lo que ocurre en el depresivo es que la retentio (el pasado) es invadido por la protentio (el futuro), con lo cual toda la temporalidad se detiene y no se llega a ninguna actualización en la presentatio (el presente). Esto explicaría todas las formas en que se presenta ese síntoma tan central de la depresión, que es la inhibición y que en investigaciones anteriores (Dörr-Zegers, 1979, 1993; DörrZegers, Enríquez \& Jara, 1971; Dörr-Zegers \& Tellenbach, 1980) hemos caracterizado como uno de los tres fenómenos fundamentales del "síndrome depresivo nuclear", pero también, por cierto, el hecho de que toda la existencia del depresivo quede detenida y al salir de la fase vuelva él al momento anterior, vale decir, al comienzo de su enfermedad, sin lograr incorporar este período a su historia vital.

Con respecto al delirio depresivo, debemos decir en primer lugar que, como ha sido reconocido desde siempre, los temas a los que se refiere no son abstrusos, extravagantes o imposibles, como ocurre en la esquizofrenia, sino temas de la vida cotidiana, como es el caso de la relación consigo mismo y particularmente con el propio cuerpo en el delirio hipocondríaco, la relación con los otros en el delirio de culpa y la relación con la sociedad en general en el delirio de ruina. Los pacientes 
afirman tener ya una enfermedad incurable (y en casos extremos, como es el síndrome de Cotard, estar ya muertos), o ser culpables de algo que puede llegar a ocurrir en el futuro (que el Servicio de Impuestos Internos encuentre una falla en su declaración anual, o de que le pueda ocurrir alguna desgracia a uno de sus hijos, etc.), o por último, afirman que ya están arruinados, lo que, aún cuando es una posibilidad en el futuro, no hay certeza alguna de que eso tenga que ocurrir. En todos estos casos se da un fenómeno exactamente inverso a lo que ocurría con los auto-reproches, a saber, que situaciones posibles en el futuro son depresión y vendría a explicar la detención e incomunicación del paciente depresivo, fenómeno descrito también por von Gebsattel (1954) y definido por él como Werdenshemmung (inhibición del llegar a ser).

\section{Intencionalidad y esquizofrenia}

El concepto más fundamental de la fenomenología es sin duda el de intencionalidad. En su significado más genérico la intencionalidad apunta al hecho que todo fenómeno psíquico se caracteriza esencialmente por estar referido a otra cosa que sí mismo. Expresado con las palabras del propio Husserl (1962), “en los simples actos de percibir estamos dirigidos a las cosas percibidas, en el recordar a las recordadas, en el pensar a los pensamientos, en el evaluar a los valores, en el desear a los objetivos y perspectivas..." (p. 287). El caso más demostrativo de la importancia de la intencionalidad es la percepción, de un objeto o de otra persona. Toda percepción es necesariamente perpectivística, vale decir, que uno percibe siempre sólo ciertos aspectos del objeto y "apercibe" el resto. La "apercepción" es una forma particular de "apresentación", fenómeno a que nos referimos con anterioridad, pero ambos se rigen por las mismas leyes. A través de la intencionalidad y por medio de los procesos de percepción / apercepción o de presentación / apresentación, el Yo constituye el objeto en su totalidad y con ello, el mundo común o koinos kosmos. En la esquizofrenia, en cambio, habría un debilitamiento de la intencionalidad, a raíz de lo cual los objetos no son (usando un neologismo) "intendidos" o intencionados como corresponde, o dicho con otras palabras, el modo de dirigirse el Yo hacia el o los objetos es insuficiente. Este fenómeno trae las siguientes consecuencias:

1. Los objetos adquieren un aspecto extraño, ajeno, surgiendo desde ellos múltiples significados que no aparecen cuando la intencionalidad funciona 
correctamente. Esto es lo que se observa característicamente en el humor delirante y en la percepción delirante.

2. El debilitamiento del arco intencional lleva, a nivel del pensamiento y del discurso, a la clásica laxitud de las asociaciones, descrita por los autores clásicos (Bleuler, 1911).

3. En ocasiones puede producirse lo que Fuchs (2005) ha descrito recientemente como "inversión de la intencionalidad". En la esquizofrenia el armónico intercambio de intencionalidades y perspectivas entre el Yo y el otro se invierte y esto ocurre de la siguiente manera: el paciente no es capaz de ponerse en la perspectiva del otro para mirar la realidad (ver Blankenburg, 1991) y por otra parte el otro, al desequilibrarse las intencionalidades, se transforma necesariamente en perseguidor e invasor, puesto que él (el otro) "intenciona" al esquizofrénico, mientras éste no está en condiciones de "intencionarlo" a él. Dicho con otras palabras, el "dirigirse a" del paciente esquizofrénico está tan debilitado que no es capaz de contrarrestar la fuerza del "dirigirse a" o intencionalidad del otro, quedando el campo de la intersubjetividad bajo el total dominio del otro. Este fenómeno que hemos descrito es el fundamento fenomenológico de ese síntoma tan frecuente en la esquizofrenia, cual es el delirio de persecución.

4. Por último, este debilitamiento de la intencionalidad, que se encuentra a la base tanto de la constitución de la intersubjetividad como de la temporalidad objetiva de estos pacientes, viene a explicar también el entorpecimiento del "camino de la vida" del esquizofrénico. Todo psiquiatra experimentado, que haya visto pacientes graves en los departamentos de agudos o seguido la evolución de otros por décadas, tendrá que recordar el vacío y la tristeza de estas vidas, donde falta el impulso y la iniciativa, donde los objetos del mundo no atraen, sino más bien invaden desde la multiplicidad de sus significaciones, donde no hay perseverancia ni por ende, logros. Pero sería un error llamar a esto pereza o apatía. El paciente esquizofrénico no puede hacer otra cosa, porque el motor mismo de la vida, que es la intencionalidad, ha fallado en la constitución del otro y del mundo común y compartido.

Ha sido tarea de la fenomenología el descubrir, en la compleja arquitectura de lo humano, el lugar de la falla que está a la base de los síntomas y síndromes que enfrentamos en la práctica clínica cotidiana. Será tarea de la neurobiología el determinar los mecanismos somáticos que subyacen a esas fallas fundamentales y de la psiquiatría biológica y de la psicofarmacología el encontrar los medicamentos que ayuden a superarlas. 


\section{Referencias}

Baeyer, W von. (1955). Der Begriff der Begegnung in der Psychiatrie. Der Nervenarzt, 26, 369-376.

Binswanger, L. (1947). Ausgewählte Vorträge und Aufsätse. I. Band. Bern, Francke Verlag, 50-74.

. (1960). Melancholie und Manie. Neske Verlag: Pfullingen.

. (1991). Perspektivität und Wahn. In: Blankenburg W (Hrsg). Wahn und Perspektivität. Stuttgart: Enke Verlag.

Bleuler, E. (1911). Dementia praecox oder die Gruppe der Schizophrenien. En: Aschaffenburg (ed). Hb der Psychiatrie. Band IV. Deuticke Verlag: Leipzig und Wien.

Dörr-Zegers, O. (1965). Zum Problem schizophrener Begegnungsweisen (mit K. Diebold). Jahrbuch für Psychologie, Psychotherapie und med. Anthropologie, 12, 23-36.

. (1979). Análisis fenomenológico de la depresividad en la melancolía y en la epilepsia. Actas Luso Españolas Neurol Psiquiat y Ciencias Afines $7\left(2^{\mathrm{a}}\right.$ Etapa): 291-304. $16(3), 22-30$.

. (1993). Fenomenología de la corporalidad depresiva. Salud Mental . (1995). Psiquiatría Antropológica. Editorial Universitaria: Santiago.

. (2002). Fenomenología de la corporalidad en la depresión delirante. Salud Mental, 25 (4), 1-9.

Döo-Zegers, O, Enríquez, G. \& Jara, C. (1971). Del análisis clínico-estadístico del síndrome depresivo a una comprensión del fenómeno de la depresividad en su contexto etiopatogénico. Rev Chil Neuropsiquiat, 10 (1), 17-39.

Dörr-Zegers, O \& Tellenbach, H. (1980). Differentialphänomenologie des depressiven Syndroms. Der Nervenarzt, 51, 113-118.

Fuchs, T. (2005). Delusional Mood and Delusional Perception - A Phenomenological Analysis. Psychopathology, 38, 133-139. 
Gebsattel, Ve Von. (1954). Die Störungen des Werdens und des Zeiterlebens im Rahmen psychiatrischer Erkrankungen. In: Prolegomena einer medizinischen Anthropologie. Springer-Verlag, Berlin . Göttingen . Heidelberg.

Husserl, E. (1962). Husserliana, Band IX, Phänomenologische Psychologie. Nijhoff, Den Haag.

. (1963). Cartesianische Meditationen und Pariser Vorträge. Husserliana I. Martinus Nijhoff, § 24: Den Haag.

Kraus, A. (1998). La especificidad del estado de ánimo y de la angustia en la melancolía. Rev Chil Neuropsiquiat, 36, 194-204.

Kranz, H. (1972). Depressionen. 2. Auflage. Banaschewski: München.

Minkowski, E. (1927). La schizophrenie: psychopathologie des schizoides et des schizophrenes. Payot: Paris.

Szilasi, W. (1959). Einführung in die Phänomenologie Edmund Husserls. Max Niemeyer Verlag: Tübingen.

Tellenbach, H. (1983). Melancholie. Springer Verlag, Berlin-Göttingen-Heidelberg, 4. Auflage. (Trabajo original publicado en 1961).

Zerssen, D Von. (1969). Objektivierende Untersuchungen zur prämorbiden Persönlichkeit endogen Depressiver. In Urban \& Schwarzenberg Verlag (orgs.) Das depressive Syndrom, München-Berlin-Wien, 183-205.

. (1970). Die prämorbide Persönlichkeit von endogen Depressiven. Confin. Psychiat. 13, S. 156.

. (1982). Personality and Affective Disorders. In Paykel ES, Handbook of Affective Disorders, Churchill Livingstone: New York. 